\title{
Renal damage induced by non-steroidal anti-inflammatory drug treatment
}

\author{
Ioana Para ${ }^{1}$, Lorena Ciumărnean ${ }^{1}$, Teodora Alexescu ${ }^{1}$, Elena-Maria Domșa $^{1}$, Mircea Vasile Milaciu ${ }^{1}$, \\ Adriana Albu ${ }^{2}$
}

Corresponding author: Lorena Ciumărnean, E-mail: lorena ciumarnean@yahoo.com

1. 4th Medical Clinic, 5th Department of Internal Medicine, Iuliu Hațieganu

University of Medicine and Pharmacy, Cluj-Napoca, Romania

2. 2th Medical Clinic, 5th Department of Internal Medicine, Iuliu Hațieganu

University of Medicine and Pharmacy, Cluj-Napoca, Romania

\begin{abstract}
Non-steroidal anti-inflammatory drugs (NSAIDs) are extensively used worldwide due to their analgesic, antipyretic and antiinflammatory effects. NSAIDs (both non-selective NSAIDs and selective cyclooxygenase-2 inhibitors) have nephrotoxic potential, particularly when used chronically. The principal mechanism of action of NSAIDs is cyclooxygenase inhibition, which prevents the conversion of arachidonic acid to prostaglandins, prostacyclins and thromboxanes. In the kidney, prostaglandins induce vasodilation and counter the action of the renin-angiotensin-aldosterone system and the sympathetic nervous system, ensuring optimal renal perfusion. Inhibition of this mechanism by NSAIDs can result in renal damage: acute kidney injury through hemodynamic mechanism, acute interstitial nephritis, glomerular disease, papillary necrosis, water and electrolyte imbalances, HTN. Chronic NSAID use may lead to chronic kidney disease.The nephrotoxic effect is reduced in young patients without renal disease or other comorbidities, but increases significantly in elderly patients with pre-existing kidney disease, nephrotic syndrome, diabetes mellitus, severe congestive heart failure, volume depletion, cirrhosis with ascites, HTN, atherosclerosis, or in patients under treatment with diuretics, angiotensin-converting enzyme inhibitors, angiotensin receptor inhibitors.
\end{abstract}

Key words: non-steroidal anti-inflammatory drugs, nephrotoxicity, kidney disease, cyclooxygenase, prostaglandins

\section{Introduction}

Non-steroidal anti-inflammatory drugs (NSAIDs) have been used since ancient times (by Assyrians, Egyptians, Greeks) for their analgesic, antipyretic and anti-inflammatory effects. Initially, they were extracted from willow bark and leaves. At the end of the 17th century, in Europe, salicin was identified as the active ingredient in the willow extract. The German company Kolbe started the mass production of salicylic acid, and in 1899 the Bayer company changed it into acetylsalicylic acid and launched it on the market under the name of aspirin $(1,2)$.

NSAIDs underwent a major development during the past century, especially after Sir John Vane, an English pharmacologist, identified their role in the inhibition of prostaglandin synthesis mediated by cyclooxygenases $(1,3,4)$. They started to be increasingly used in the treatment of a wide range of conditions, from minor pain and fever to osteoarticular disorders (acute and chronic) or inflammatory intestinal diseases. With their extensive use, adverse reactions started to appear: digestive hemorrhage, ulcer, arterial hypertension, platelet dysfunction syndrome, cardiovascular adverse reactions and renal damage $(1,5,6,7)$.

Currently, NSAIDs are among the most widely used drugs globally, and most of them can be bought over the counter. The most frequent users are those with chronic pain associated with rheumatic diseases (rheumatoid arthritis, osteoarthritis or other musculoskeletal disorders).

The pharmacological effect of NSAIDs depends on the dose and the duration of treatment. Prolonged use leads to organ damage, renal involvement ranking second in terms of frequency $(8,9,10)$.

\section{The mechanism of action of NSAIDs}

The principal mechanism of action of NSAIDs is central and peripheral cyclooxygenase (COX) inhibition, which interferes with the conversion of arachidonic acid to prostaglandins (PG), prostacyclins and thromboxanes. Prostaglandins have a vasodilator effect, extremely important for the maintenance of preglomerular resistance, glomerular filtration rate and renal blood flow $(1,5,11,12)$.

There are two COX isoforms, COX-1 and COX-2, which act at different levels. The COX-1 isoform has a role in regulating physiological processes and maintaining homeostasis; therefore, it is present in the majority of cells and tissues (endothelium, monocytes, platelets, renal collecting ducts, gastrointestinal tract, seminal vesicles). $\mathrm{COX}-2$ is activated by inflammation and pro-inflammatory cytokines in the vascular endothelium, osteoclasts and macrophages $(1,5,7,13,14)$. NSAIDs can be 
classified into four categories, depending on their selectivity in inhibiting COX-1 and/or COX-2 $(1,5,15)$ (Table 1).

Table 1. Classification of NSAIDs depending on inhibition of COX-1 and/or COX-2 (15).

\begin{tabular}{|l|l|l|}
\hline Class & Properties & Examples \\
\hline Group 1 & $\begin{array}{l}\text { NSAIDs that } \\
\text { inhibit both } \\
\text { COX1 and COX- } \\
2\end{array}$ & $\begin{array}{l}\text { Aspirin, ibuprofen, } \\
\text { ketoprofen, diclofenac, } \\
\text { indomethacin, naproxen, } \\
\text { piroxicam }\end{array}$ \\
\hline Group 2 & $\begin{array}{l}\text { NSAIDs that are } \\
\text { preferential COX- } \\
\text { 2 inhibitors }\end{array}$ & $\begin{array}{l}\text { Celecoxib, etodolac, } \\
\text { meloxicam, nimesulide }\end{array}$ \\
\hline Group 3 & $\begin{array}{l}\text { NSAIDs that are } \\
\text { highly selective } \\
\text { COX-2 inhibitors }\end{array}$ & Rofecoxib, NS-398 \\
\hline Group 4 & $\begin{array}{l}\text { NSAIDs that are } \\
\text { weak inhibitors of } \\
\text { both isoforms }\end{array}$ & $\begin{array}{l}\text { 5-aminosalicylic acid, } \\
\text { sodium salicylate, } \\
\text { nabumetone, sulfasalazine }\end{array}$ \\
\hline
\end{tabular}

The majority of adverse effects are related to COX-1 inhibition. In the kidney, COX-1 has an important role in maintaining glomerular filtration, which is why the renal adverse effects of using non-selective NSAIDs are greater in patients with pre-existing renal involvement. The action of COX-2 is associated with renal water and electrolyte balance; thus, renal adverse effects are enhanced by dehydration, decreased renal perfusion or pre-existing renal damage $(5,14,16,17)$.

\section{The mechanisms by which NSAIDs can induce} renal damage

The kidneys achieve complex functions: they maintain homeostasis (fluid volume and osmolarity, water, electrolyte and acid-base balance), excrete metabolic end products, metabolize and excrete a series of exogenous substances, including drugs. Prostaglandin synthesis plays an important role in ensuring the filtration function by maintaining the glomerular filtration rate and renal homeostasis $(1,5,18,19)$.

NSAIDs inhibit coenzymes COX-1 and/or COX-2 and implicitly, the formation of prostaglandins. In the kidney, prostaglandins (especially prostacyclin, PGE2, PGD2) have a vasodilator action on the afferent arteriole, increasing renal perfusion, with the distribution of blood flow from the renal cortex to the medulla. Furthermore, vasodilation has a negative feedback effect on the renin-angiotensin-aldosterone system and the sympathetic nervous system, ensuring adequate blood flow in the kidney. NSAIDs, through inhibition of PG synthesis, can lead to acute vasoconstriction and acute kidney injury
$(5,10,18,19,20)$.

In addition to its vasodilator action, PGE2 inhibits the transport of sodium and chloride in the ascending limb of the loop of Henle and in the collecting duct, resulting in natriuresis. PGE2 also has an antagonist effect on antidiuretic hormone receptors, favoring diuresis. The inhibition of PGE2 production by NSAIDs leads to sodium and water retention, with the development of edema and arterial hypertension $(5,19,21,22,23,24)$.

Clinical renal syndromes associated with the use of NSAIDs NSAID treatment may affect cells in all nephron components through various mechanisms $(1,9,25)$ (Table 2).

Table 2. Renal syndromes induced by NSAIDs $(1,9)$.

\begin{tabular}{|c|c|}
\hline Acute kidney injury & $\begin{array}{l}\text { Hemodynamically mediated } \\
\text { Acute tubular necrosis }\end{array}$ \\
\hline \multicolumn{2}{|l|}{ Acute interstitial nephritis } \\
\hline Glomerular disease & $\begin{array}{l}\text { Minimal change disease } \\
\text { Membranous nephropathy }\end{array}$ \\
\hline \multicolumn{2}{|l|}{ Papillary necrosis } \\
\hline Electrolyte abnormalities & $\begin{array}{l}\text { Hyperkalemia } \\
\text { Renal tubular acidosis } \\
\text { Hyponatremia }\end{array}$ \\
\hline \multicolumn{2}{|l|}{ Hypertension/edema } \\
\hline $\begin{array}{l}\text { Analgesic } \\
\text { nephropathy/chronic } \\
\text { tubulointerstitial nephritis }\end{array}$ & \\
\hline
\end{tabular}

Acute kidney injury (AKI)

The most frequent form of AKI induced by NSAIDs is hemodynamically mediated, and may occur following both non-selective NSAIDs and selective NSAIDs. AKI develops due to an alteration of intrarenal microcirculation and implicitly, of glomerular filtration by inhibition of PG synthesis $(9,25,26,27)$.

Under euvolemic conditions, basal prostaglandin levels are not very high; this is why intrarenal circulation does not depend on these substances to maintain the glomerular filtration rate. Consequently, under these circumstances, prostaglandin synthesis inhibition induced by NSAIDs rarely affects renal circulation and function. In contrast, under conditions of volume depletion, severe hypotension, hemodynamic instability, prostaglandin synthesis is significantly increased to counterbalance vasoconstriction induced by angiotensin II, norepinephrine, endothelin and vasopressin, and to ensure adequate renal perfusion, with the maintenance of glomerular filtration and the reduction of ischemia. In these patients, prostaglandin synthesis inhibition by NSAIDs leads to a decrease in 
the glomerular filtration rate, acute ischemia that increases the risk of acute tubular necrosis, an enhancement of vascular tone with antidiuretic and antinatriuretic effects $(1,5,10,28,29)$.

AKI induced by NSAIDs is not frequent, but its incidence increases significantly in patients at risk such as: patients with volume and sodium depletion, congestive heart failure, cirrhosis with ascites, nephrotic syndrome, chronic kidney disease, arterial hypertension, diabetes mellitus, over 65 years of age (especially in the case of comorbidities) $(28,29,30,31)$. Also, there is an increased risk for AKI in the case of the association of NSAIDs with angiotensin-converting enzyme inhibitors (ACEIs), angiotensin receptor blockers (ARBs) or diuretics $(1,5,7,10,29,32,33)$.

AKI clinically manifests with oliguria and an elevation in serum creatinine levels, which occur 3-7 days after administration of NSAIDs. Proteinuria, if present, is lower than $500 \mathrm{mg} /$ day. Absence of hematuria and proteinuria in the presence of granular and epithelial cylinders is specific to ischemic injury. AKI treatment consists of immediate interruption of NSAIDs and volume repletion (if needed). Otherwise, the management of AKI is identical to that of AKI of other causes $(1,9,10,28)$.

\section{Acute interstitial nephritis}

Acute interstitial nephritis is responsible for about $15 \%$ of AKI cases. It can have multiple causes, but is most frequently induced by drugs, among which NSAIDs play an important role $(1,10,25,34,35)$.

Pathogenetically, a type IV delayed hypersensitivity reaction occurs, which is mediated by $\mathrm{T}$ helper 2 lymphocytes. Renal function alteration develops through acute inflammation and edema of the renal interstitium, associated with tubulitis and lymphocyte and eosinophil infiltration $(35,36,37)$.

AIN induced by NSAIDs has several clinical particularities: it occurs after a long treatment duration, 6-18 months (for both non-selective and COX-2 selective NSAIDs), and patients do not present fever, rash or eosinophilia (which are probably masked by the anti-inflammatory effects of NSAIDs) $(1,25,28,35)$.

Despite AKI that can be severe, treatment interruption is followed in the majority of cases by renal function restoration $(1,25,35)$.

\section{Glomerular disease}

Glomerular disease induced by NSAIDs (selective and non-selective) is represented by minimal change disease (MCD) with nephrotic syndrome and rarely, by membranous nephropathy (MN) $(25,38,39)$. Pathogenesis is not completely understood and is more probably due to an alteration of immune response secondary to $\mathrm{COX}$ inhibition than to direct toxic cellular effects. MCD can be associated with AIN. Interruption of treatment with NSAIDs usually results in remission of glomerular disease. If nephrotic syndrome persists after interruption of NSAID treatment, corticotherapy is associated, with favorable results $(1,9,25,38)$.

\section{Papillary necrosis}

NSAIDs may cause acute, subacute or chronic papillary necrosis, which constitutes analgesic nephropathy. PG synthesis inhibition by NSAIDs leads to a diminution of blood flow in the renal medulla and papilla, with ischemia and even papillary necrosis. Administration of NSAIDs under volume depletion conditions (after vomiting, diarrhea, sepsis, diuretics, insufficient intake) increases the risk of acute tubular necrosis $(1,40,41,42)$.

Clinically, there is an alteration of the urine concentration capacity, sterile pyuria, microscopic hematuria and low-level proteinuria, with an increase in serum creatinine values. Computed tomography can be useful for diagnosis $(1,29,42)$.

Prognosis depends on the time of diagnosis and on the time of treatment interruption. Continuing NSAID treatment can lead to end-stage chronic kidney disease $(1,29,41)$.

\section{Alteration of water and electrolyte balance by NSAIDs}

Hyperkalemia occurs by two mechanisms. In the first place, it inhibits renin secretion mediated by PG, with the development of hyporeninemic hypoaldosteronism, which results in decreased potassium secretion in the collecting duct cells with hyperkalemia. In the second place, NSAIDs induce AKI with a reduction of glomerular filtration.

The diminution of glomerular filtration leads to a decrease in sodium concentration in the distal nephron, with a decrease in potassium secretion at this level. Hyperkalemia is more severe in patients with intravascular volume depletion, heart failure, or patients receiving NSAIDs associated with ACEI, ARBs or aldosterone blockers $(1,10,29,30,43)$.

Renal tubular acidosis occurs due to prolonged hyperkalemia, which leads to a decrease in renal ammonium ion excretion in the distal nephron, with type 4 renal tubular acidosis, a form of 
hyperchloremic metabolic acidosis $(1,10,43)$.

Hyponatremia develops because of PG secretion inhibition by NSAIDs and the disappearance of their regulatory effect on the antidiuretic hormone, with increased water reabsorption and dilution hyponatremia. The decrease in the glomerular filtration rate also favors water retention and hyponatremia $(1,10,43)$.

\section{Arterial hypertension}

The inhibition of PG production by NSAIDs results in sodium and water retention, with the development of edema and increased blood pressure values. This effect is more intense in patients with pre-existing HTN, heart failure, chronic kidney disease or cirrhosis $(1,10,28,44)$.

\section{NSAIDs and chronic kidney disease}

Both types of NSAIDs (selective and non-selective) inhibit intrarenal vasodilation mediated by PG, with a decrease in renal perfusion and an alteration of the glomerular filtration rate, which is why they should be avoided in patients with a glomerular filtration rate lower than $60 \mathrm{ml} / \mathrm{min}$. In these patients, even small doses of NSAIDs can precipitate the development of renal failure with all the mechanisms described above. Moreover, these patients usually receive treatment with ACEIs, ARBs or diuretics for HTN, proteinuria or volume control, which in association with NSAIDs increase even more the risk of rapid alteration of the renal function $(1,5,9,10,45)$.

\section{Conclusions}

NSAIDs, both non-selective and selective (including coxibs), can induce renal damage. The risk of renal involvement is lower in young patients without associated diseases, but increases significantly in elderly patients (who usually have several associated diseases requiring drug associations and have reduced renal hemodynamics), with pre-existing kidney disease, nephrotic syndrome, diabetes mellitus, severe congestive heart failure, volume depletion, cirrhosis with ascites, HTN, atherosclerosis, or in patients under treatment with diuretics, ACEIs or ARBs. This is why careful monitoring of the renal function during prolonged NSAID treatment is necessary, particularly in patients with risk factors. Early identification of renal damage and interruption of treatment with NSAIDs lead in the majority of cases to the recovery of renal function or at least, to its improvement.

\section{Declaration of conflict of interests}

The author does not have any financial interest involving the companies and/or materials mentioned in this article.

\section{References}

1. Rahman S, Malcoun A. Nonsteroidal Antiiflamatory Drugs, Cyclooxygenase- 2 and the Kidneys. Prim Care Clin Office Pract. 2014; 41:803-821.

2. Vane JR. The fight against rheumatism: from willow bark to COX-1 sparing drugs. J Physiol Pharmacol. 2000; 51:573-586.

3. Vane JR. Inhibition of prostaglandin synthesis as a mechanism of action for aspirin-like drugs. Nat New Biol. 1971; 43:232-235.

4. Vane JR. The mode of action of aspirin and similar compounds. J Allergy Clin Immunol. 1976; 58:691-712.

5. Cavalcanti Lucas GN, Carneiro Leitao AC, Alencar RL, Fagundes Xavier RM, De Francesco Daher E, Bezerra da Silva Junior G. Pathophysiological aspects of nephropathy caused by non-steroidal anti-inflammatory drugs. J Bras Nephrol. 2018; Sep 21, DOI: 10.1590/2175-8239-JBN2018-0107

6. Harirforoosh S, Asghar W, Jamali F. Adverse effects of nonsteroidal anti-inflammatory drugs: an update of gastrointestinal, cardiovascular and renal complications. J Pharm Pharm Sci. 2013; 16:821-847.

7. Curiel RV, Katz JD, Mitigating the Cardiovascular and Renal Effects of NSAIDs. Pain Medicine. 2013; 14:S23S28.

8.Wehling M,. Non-steroidal anti-inflammatory drugs use in chronic pain conditions with special emphasis on the elderly and patients with relevant comorbodities: management and mitigation of risks and adverse effects. Eur J Clin Pharmacol. 2014;70:1159-1172.

9. Perazella MA. Drug-Induced nephropathy: an update. Expert Opin Drug Saf. 2005;4:689-706.

10. Perazella MA, Shirali A. Kidney Disease Caused by Therapeutic Agents. In: Gilbert SJ, Weiner DE, editors. National Kidney Foundation's Primer on Kidney Diseases. 6th edition. Philadelphia: Elsevier; 2014:326336.

11. Harris, R. C. Physiologic and pathophysiologic roles of cyclooxygenase-2 in the kidney. Trans Am Clin Climatol Assoc. 2013;124:139-151.

12. Harris, R. C., \& Zhang, M. Z. Cyclooxygenase metabolites in the kidney. Compr Physiol.2011; 1:1729_ 1758.

13. Pountos I, Georgouli T, Bird H, Giannoudis PV. Nonsteroidalanti-inflammatory drugs: prostaglandins, indications and side effects. Int $\mathrm{J}$ Interferon Cytokine Mediator Res.2011;3:19-27

14. Rang HP, Dale MM. Farmacologia. 8th ed. Rio de Janeiro: Elsevier; 2016.

15. Rao PN, Knaus EE. Evolution of nonsteroidal antiinflammatory drugs (NSAIDs): cyclooxigenase(COX)inhibition and beyond. J Pharm 
Pharm Sci.2008;11(2):81s-110s.

16. Grosser T, Fries S, FitzGerald GA. Biological basis for cardiovascular consequences of $\mathrm{COX}-2$ inhibition: Therapeutic challenges and opportunities. J Clin Inves. 2006;116:4-15.

17. Rios A, Vargas-Robles H, Gamez-Mendez AM,Escalante B. Cyclooxygenase-2 and kidney failure. Prostaglandins Other Lipid Mediat. 2012;98 (3-4):86-90.

18. Green T, Gonzalez AA, Mitchell KD, Navar LG. The complex interplay between cyclooxygenase-2 and angiotensin II in regulating kidney function. Curr Opin Nephrol Hypertens. 2012;21:7-14.

19. Ejaz P, Bhojani K, Joshi VR. NSAIDs and kidney. J Assoc Physicians India. 2004; 52:632-640.

20. Burukoglu D, Baycu C, Taplamacloglu F, Sahin E, BekturE. Effects of nonsteroidal anti-inflammatory meloxicam on stomach, kidney and liver of rats. Toxicol Ind Health. 2016;32:980-986.

21. Brater DC. Anti-Inflammatory Agents and Renal Function. Seminars in Arthritis and Rheumatism. 2002; 32 (Suppl 1): 33-42.

22. Harris RC. Cyclooxygenase-2 inhibition and renal physiology. Am J Cardiol. 2002;89(6A):10D-17D.

23. Gonzalez AA, Cespedes C, Villanueva S, et al.Eprostanoid-1 receptor regulates renal medullary alphaENaC in rats infused with angiotensin II. Biochem Biophys Res Commun. 2009;389:372-377.

24. Hörl Wh. Nonsteroidal Anti-Inflammatory Drugs and the Kidney. Pharmaceuticals (Basel). 2011;3:2291-2321.

25. Paueksakon P, Fogo AB. Drug-induced nephropathies. Histopathology. 2017;70:94-108.

26. Luciano RL, Perazella MA. Drug-Induced Acute Kidney Injury. In: SS Waikar et al, editors. Core Concepts in Acute Kidney Injury.Springer. 2018; 145-163.

27. Bellomo R, Kellum JA, Ronco C. Acute kidney injury. Lancet 2012;380:756-66.

28. Weir MA, Ronco C, House AA. Antiinflammatory Drugs and the Kidney. In: Ronco C, Bellomo R, Kellum JA, Ricci Z, editors. Critical Care Nephrology. 3th edition. Philadelphia: Elsevier; 2019;1306-1309.

29. Nolin TD, Himmelfarb J. Mechanism of Drug-Induced Nephrotoxicity. In: Uetrecht J, editor. Adverse Drug Reactions. Handbook of Experimental pharmacology. Springer-Verlag Berlin Heidelberg. 2010;111-130.

30. Dogaru G, Motricală M, Ákos M, Rus V. Effects of mineral water from spring 3 in Băile Tuşnad on experimentally induced alcoholic liver disease.Balneo Research Journal. 2017;8(3):125-128.

31. Dogaru G, Motricală M, Ákos M, Rus V. An experimental study regarding the biological effects of mineral water from spring 3 in Băile Tuşnad on some organs after ethyl alcohol administration. Balneo Research Journal. 2016;7(1):23-28.

32. Prieto-Garcia L, Pericacho M, Sancho-Martinez SM, Sanchez A, Martinez-Salgado C, Lopez-Novoa JM,
Lopez-Hernandez FJ. Mechanisms of triple whammy acute kidney injury. Pharmacology \& Therapeutics. 2016;167:132-145.

33. Dreischulte T, Morales DR, Bell S, Guthrie B. Combined use of nonsteroidal anti-inflammatory drugs with diuretics and/or renin-angiotensin system inhibitors in the community increases the risk of acute kidney injury. Kidney Int 2015;88:396-403.

34. Brewster UC, Rastegar A. Acute Interstitial Nephritis. In: Gilbert SJ, Weiner DE, editors. National Kidney Foundation's Primer on Kidney Diseases. 6th edition. Philadelphia: Elsevier; 2014;312-317.

35. Krishnan N, Perazella MA. Drug-induced Acute Interstitial Nephritis. IJKD. 2015;9:3-13.

36. Chang C, Gershwin ME. Drugs and autoimmunity-a contemporary review and mechanistic approach. J Autoimmun. 2010;34:J266-J275.

37. Adam J, Pichler WJ, Yerly D. Delayed drug hypersensitivity: models of T-cell stimulation. Br J Clin Pharmacol. 2011;71:701-707.

38. Radhakrishnan J, Perazella MA. Drug-Induced Glomerular Disease: Attention Required. Clin J Am Soc Nephrol. 2015;10:1287-1290.

39. Nawaz FA, Larsen CP, Troxell ML. Membranous nephropathy and nonsteroidal anti-inflammatory agents. Am J Kidney Dis. 2013;62(5):1012-1017.

40. Brix AE. Renal papillary necrosis. Toxicol Pathol. 2002;30:672-674.

41. Braden GL, O'Shea MH, Mulhern JG. Tubulointerstial diseases. Am J Kidney Dis. 2005;46:560-572.

42. Silva FG. Chemical-induced nephropathy: a review of the renal tubulointerstitial lesions in humans. Toxicol Pathol.2004;32(Suppl 2):71-84.

43. Kim S, Joo KW. Electrolyte and Acid-base disturbances associated with non-steroidal antiinflammatory drugs. Electrolyte Blood Press 2007;5:116125.

44.Frishman WH. Effects of nonsteroidal antiinflammatory drug therapy on blood pressure and peripheral edema. Am J Cardiol. 2002;89(6A):18D-25D.

45. Chang Y-K, Liu J-S, Hsu Y-H, et al. Increased risk of end-stage renal disease (ESRD) requiring chronic dialysis is associated with use of nonsteroidal anti-inflammatory drugs (NSAIDs): nation $\neg$ wide case-crossover study. Medicine (Baltimore). 2015;94(38): e1362. 